\title{
A Model of Energy Deposition of Energetic Electrons and EUV Emission in the Jovian and Saturnian Atmospheres and Implications
}

\author{
J.-Cl. GÉrard ${ }^{1}$ ANd V. Singh \\ Institut d'Astrophysique, Université de Liège, B-4200 Cointe-Liège, Belgium
}

\begin{abstract}
A model of the interaction between incident electron precipitation and $\mathrm{H}_{2}$ atmospheres is described. The local degraded primary and secondary electron energy distributions are calculated by using the continuous slowing down approximation. The altitude distribution of the ionization rate and various $\mathrm{H}$ and EUV $\mathrm{H}_{2}$ emissions are calculated for four different incident electron spectra. A total EUV $\mathrm{H}_{2}$ emission efficiency of $10.6 \mathrm{kR} /$ incident $\mathrm{erg} \mathrm{cm}^{-2} \mathrm{~s}^{-1}$ is obtained for a pure $\mathrm{H}_{2}$ atmosphere. Comparison with the Voyager Jupiter observations indicates that an incident energy flux of about 8 ergs cm$~_{-2}^{-1} \mathrm{~s}^{-1}$ was present at the time of the encounter if the emission is located in an $\mathrm{H}_{2}$-dominated region. The local

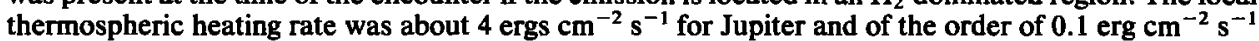
for Saturn. A globally averaged atomic hydrogen production rate of $\sim 1 \times 10^{10}$ atoms $/ \mathrm{cm}^{2} \mathrm{~s}^{-1}$ is induced by the Jovian auroral electron precipitation, largely exceeding the solar EUV dissociation rate.
\end{abstract}

\section{INTRODUCTION}

The existence of optical emissions resulting from the interaction with the atmosphere of charged particles accelerated in the magnetosphere of giant planets has been suspected by analogy with the earth's polar aurora. Various observers have searched for the presence of emission lines from atomic hydrogen on the nightside of the planet. Schwitters [1968] and Hunter [1969] claimed to have observed Balmer $\alpha$ emission with an intensity of a few kiloRayleighs. However, a search for $\mathrm{H} \alpha$ by Dulk et al. [1970] gave inconclusive results and put an upper limit of $10 \mathrm{kR}$ on the apparent emission rate. More recently, the discovery of an extensive magnetosphere around Jupiter by the Pioneer 10 and 11 probes and the discovery of the Io plasma torus confirmed the possibility that optical emissions may be produced by the precipitation of charged particles into the Jovian polar atmosphere.

Localized enhancements of the Lyman $\alpha$ 1216- $\AA$ line were observed by Atreya et al. [1977] by using the Copernicus satellite. They detected a bright spot of emission exceeding $100 \mathrm{kR}$ located at the foot of the Io plasma torus at latitudes between $65^{\circ}$ and $70^{\circ}$. Spatially resolved spectra of the polar regions were obtained with the International Ultraviolet Explorer satellite (IUE) by Clarke et al. [1980] at 11resolution. They reported variations in brightness of the $\mathrm{H}_{2}$ Lyman and Werner band emissions by more than an order of magnitude as well as changes in the apparent Ly $\alpha / \mathrm{H}_{2}$ bands ratio. The strongest aurorae were observed when the magnetic pole faced toward the earth. A luminosity observed near the dark limb by the imaging experiment on Voyager 1 has been identified as auroral arcs by Cook et al. [1981]. The emission is likely due to the $\mathrm{H}_{2}$ Fulcher $\alpha$ bands and a ${ }^{3} \Sigma-$ $b^{3} \Sigma$ continuum with a total intensity of about $20 \mathrm{kR}$.

Extensive observations of the dayside and nightside of

\footnotetext{
${ }^{1}$ Visiting Fellow at the Laboratory for Atmospheric and Space Physics, University of Colorado at Boulder, Boulder, Colorado 80309
}

Copyright 1982 by the American Geophysical Union.

Paper number 2 A0186.

0148-0227/82/002A-0186\$05.00
Jupiter were made by the ultraviolet spectrometer (UVS) on board Voyager 1 [Broadfoot et al., 1979] and Voyager 2 [Sandel et al., 1979]. These observations have been reviewed and discussed by Broadfoot et al. [1981a]. Spectra of the nightside were obtained between 500 and $1700 \AA$ with a resolution of $33 \AA$. They revealed the presence in the polar regions of the $\mathrm{H}_{2} B^{1} \Sigma \rightarrow X^{1} \Sigma$ and $C^{1} \Pi \rightarrow X^{1} \Sigma$ systems besides the Ly $\alpha$ line. The total measured intensity is of the order of $80 \mathrm{kR}$ for the total $\mathrm{H}_{2}$ emission and $40 \mathrm{kR}$ for $\mathrm{Ly} \alpha$. However, large uncertainties in the actual emission rate are due to the finite field of view of the instrument and possible temporal variation. An asymmetry between the intensities measured in the northern and southern auroral zones was also observed but in opposite directions between the Voyager 1 and Voyager 2 measurements. The location of this auroral zone $\left(\cong 65^{\circ}\right)$ coincides approximately with the foot of the magnetic field lines which map the plasma torus into the Jupiter atmosphere, although a contribution from the magnetotail particles cannot be excluded. At low latitudes, an upper limit of $0.5 \mathrm{kR}$ was set on the emission rate of the $\mathrm{H}_{2}$ bands [McConnell et al., 1980], but a Ly $\alpha$ emission of 400$500 \mathrm{R}$ exhibiting a marked longitudinal dependence was observed by Voyager 2. Recent IUE spectra of the Jovian aurora in the range $1100-1700 \AA$ have been obtained by Durrance et al. [1982] with improved signal-to-noise ratio. The auroral spectrum shows the same spectral distribution as laboratory spectra of discharge-excited $\mathrm{H}_{2}$. In particular, the $\mathrm{H}_{2}$ Lyman bands intensity distribution exhibits no absorption by $\mathrm{CH}_{4}$, whose cross section steeply increases below $1400 \AA$. An upper limit of a fraction of optical depth corresponding to a column density of $\sim 2 \times 10^{17} \mathrm{~cm}^{-2}$ can be put on the slant density of $\mathrm{CH}_{4}$ above the emission.

The identity of the particles which interact with the planet is not yet firmly established and electrons, protons, or heavy ions from the plasma torus of the magnetotail are potential candidates. Goertz [1980] has argued that protons rather than electrons are responsible for the excitation of the observed airglow. By examining the observed $\mathrm{H}_{2}$ bands/Ly $\alpha$ intensity ratio and the uniformity of Ly $\alpha$ across the disc, McConnell et al. [1980] have deduced that the nonpolar precipitation responsible for the global $500 \mathrm{R} \mathrm{Ly} \alpha$ emission 
is made of soft electrons or protons depositing their energy at levels where the atomic hydrogen density exceeds the $\mathrm{H}_{2}$ density.

Crude observations of the Ly $\alpha$ latitudinal distribution of the nightside of Saturn were made with the UV photometer on Pioneer 11 [Judge et al., 1980]. A weak enhancement of the emission rate was observed in the polar regions, suggesting the possibility of auroral precipitation. This interpretation was confirmed by the Voyager 1 encounter with Saturn in 1980. The UVS experiment detected the presence of the $\mathrm{H}_{2}$ bands and Ly $\alpha$ emission with emission rates varying in time between 2 and $15 \mathrm{kR}$ and 1 and $20 \mathrm{kR}$, respectively [Broadfoot et al., 1981 b]. The EUV spectrum is very similar to the Jovian emission and the auroral zone extends from $78^{\circ}$ to $81.5^{\circ}$ latitude. This location indicates that the magnetotail activity is responsible for the precipitation of charged particles in the auroral zones. Observations with the IUE satellite [Clarke et al., 1981] have also shown Ly $\alpha$ enhancements in the Saturn polar regions.

The excitation of the Jovian aurora by electron impact was first studied by Heaps et al. [1973]. They calculated the altitude distribution and integrated column intensities for the main $\mathrm{H}_{2}$ and $\mathrm{H}$ emissions. Their study assumed a cold exosphere $\left(T=190^{\circ} \mathrm{K}\right)$ and a high density at mesopause of 4 $\times 10^{14} \mathrm{~cm}^{-3}$. Their computations were based on cross sections for the $\mathrm{H}_{2}$ excited states whose values have been revised since. In this study, we have developed a model of the interaction of an incident electron beam with $\mathrm{H}_{2}$ model atmospheres based on recent Voyager measurements. The ionization rate and the local energy spectrum are calculated by using the continuous slow-down approximation. The altitude profiles of the Werner and Lyman bands as well as the Balmer and Ly $\alpha$ lines are deduced on the basis of recent cross sections. By comparing the integrated intensities with the observations, the total energy fluxes into the Jovian and Saturnian auroral zones are determined for the case of electron precipitation. The particle heat input into the highlatitude atmospheres and the heating efficiency are evaluated. The importance of the particle-induced dissociation of $\mathrm{H}_{2}$ as a source of atomic hydrogen is discussed.

\section{IONIZATION AND EXCITATION OF AN $\mathrm{H}_{2}$ Atmosphere by Primary Electrons}

The method adopted in this model is based upon the continuous slowing down approximation, which assumes that it is possible to replace discrete energy losses by a continuous function. The details of this method have been given elsewhere [Edgar et al., 1975] and will not be developed here. In this approximation, the number of ion pairs created in a gas $j$ at an altitude $z$ per unit time in a unit volume is given by

$$
q_{j}(z)=\frac{1}{\Delta E_{j}} \int_{\Omega} d \Omega \int_{E_{0}} \Phi\left(E_{0}\right)\left[-\frac{d E_{0}}{d x}\right]_{j} d E_{0}
$$

where $q_{j}(z)$ is the volume ionization rate at altitude $z, \Omega$ is the solid angle, $[-d E / d x]_{j}$ is the stopping power, $\Delta E_{j}$ is the average energy expanded in creating an electron ion pair, and $\Phi\left(E_{0}\right)$ is the initial differential electron flux outside the atmosphere.

For a given pitch angle $\theta$ the electron will travel over a differential path $\Delta z \sec \theta$. The stopping power as a function of $z$ becomes

$$
-\left[\frac{d E_{0}}{d z}\right]_{j}=\frac{n_{j}(z) L_{j}\left(E_{0}\right)}{\cos \theta}
$$

where $L\left(E_{0}\right)$ is the total loss function in gas $j$ at energy $E_{0}$. In a mixture of $\mathrm{H}_{2}$ and $\mathrm{H}$, the stopping power is given by the following expression

$$
-\frac{d E_{0}}{d z}=n(z) L_{T}\left(E_{0}, z\right) / \cos \theta
$$

where

$$
L_{T}\left(E_{0}, z\right)=\sum_{j} f_{j}(z) L_{j}\left(E_{0}\right)
$$

$n(z)$ is the total number density, and $f_{j}(z)$ the fractional abundance of gas $j$ at altitude $z$. In the separated atom model, the loss function in $\mathrm{H}$ is half the $\mathrm{H}_{2}$ loss function. Consequently, the total loss function may be written

$$
L_{T}\left(E_{0}, z\right)=\frac{1}{2}\left(1+f_{\mathrm{H}_{2}}\right) L_{\mathrm{H}_{2}}\left(E_{0}\right)=F(z) L_{\mathrm{H}_{2}}\left(E_{0}\right)
$$

Cravens et al. [1975] have compiled the electron loss function in $\mathrm{H}_{2}$ between $1 \mathrm{eV}$ and $10 \mathrm{keV}$. Above $13 \mathrm{eV}$, we represent their loss function approximately in an analytical form similar to that used by Peterson and Green [1968] to characterize a number of atmospheric gases:

$$
L_{\mathrm{H}_{2}}\left(E_{0}\right)=L_{0}\left[\left(\frac{E_{0}}{A}\right)^{P}+\left(\frac{E_{0}}{B}\right)^{M}+\left(\frac{E_{0}}{C}\right)^{N}\right]^{-1}
$$

with

$$
L_{0}=\frac{q_{0} Z}{2 R_{e}}
$$

where $q_{0}=6.514 \times 10^{-14} \mathrm{eV}^{2} \mathrm{~cm}^{2}, R_{e}=13.6 \mathrm{eV}$. The parameters $Z, A, B, C, P, M$, and $N$ are listed in Table 1 . Below $13 \mathrm{eV}$, the loss function is taken numerically from Cravens et al. [1975].

After substituting the above expression for $L_{\mathrm{H}_{2}}\left(E_{0}\right)$ into formula (3) and $L_{T}\left(E_{0}, z\right)$ into (2), equation (2) may be integrated to yield

$$
\begin{aligned}
& \frac{E^{P+1}}{A^{P}(P+1)}+\frac{E^{M+1}}{B^{M}(M+1)}+\frac{E^{N+1}}{C^{N}(N+1)} \\
&= \frac{E_{0}^{P+1}}{A^{P}(P+1)}+\frac{E_{0}^{M+1}}{B^{M}(M+1)}+\frac{E_{0}^{N+1}}{C^{N}(N+1)}-\frac{L_{0}}{\cos \theta} \\
& \cdot \int_{\infty}^{z} F\left(z^{\prime}\right) n\left(z^{\prime}\right) d z^{\prime}
\end{aligned}
$$

where $n\left(z^{\prime}\right)$ is the number density at altitude $z^{\prime}$ and $E$ is the local energy at altitude $z$ of an electron of initial energy $E_{0}$. The value of $E$ may be obtained by numerically inverting equation (4). Once the value of $E$ is known, it is then possible to evaluate the stopping power and the volume ionization integral (1). Assuming an isotropic pitch angle distribution over one hemisphere for the incident electron flux, we interchange the order of integration in equation (1) to get

$$
q_{j}(z)=\frac{2 \pi n(z) f_{j}}{\Delta E_{j}} \int_{E_{0}} \Phi\left(E_{0}\right) d E_{0} \int_{0}^{\theta_{\max }} L_{j}\left[E\left(E_{0}, z, \theta\right)\right] \tan \theta d \theta
$$


TABLE 1. Parameters for the $\mathrm{H}_{2}$ Loss Function

\begin{tabular}{ccccccc}
\hline$Z$ & $P$ & $M$ & $N$ & $A$ & $B$ & $C$ \\
\hline 5.03 & 0.823 & 0.125 & -0.96 & 100.0 & 60.0 & 140.0 \\
\hline
\end{tabular}

with

$$
\begin{aligned}
\cos \theta_{\max }=\left[L_{0}\right. & \left.\int_{\infty}^{z} F\left(z^{\prime}\right) n\left(z^{\prime}\right) d z^{\prime}\right] /\left[\left(E_{0}{ }^{P+1} / A^{P}(P+1)\right)\right. \\
& \left.+\left(E_{0}{ }^{M+1} / B^{M}(M+1)\right)+\left(E_{0}{ }^{N+1} / C^{N}(N+1)\right)\right]
\end{aligned}
$$

We have evaluated equation (5) numerically with all the parameters described in the text. The average value of $\Delta E$ is $37 \mathrm{eV}$ for $\mathrm{H}_{2}$ [Valentine and Curran, 1958].

The volume excitation rate $\boldsymbol{P}_{i}(z)$ due to primary electrons at altitude $z$ for $i$ th state can be obtained from the following expression:

$$
P_{i}(z)=\int_{E_{0}} \Phi\left(E_{0}\right) d E_{0} \int_{\Omega} \frac{d J_{i}}{d E}\left[-\frac{d E}{d x}\right] d \Omega
$$

which can be evaluated in the same manner as the volume ionization rate. The parameter required to compute the volume excitation rate is the population $J_{i}$ of that state, which is the total number of excitations into the $i$ th state along the path of an incident electron. The population of $i$ th state is

$$
J_{i}\left(E_{0}\right)=\int_{E_{i}^{\text {in }}}^{E_{0}} \frac{\sigma_{i}\left(E^{\prime}\right)}{L\left(E^{\prime}\right)} d E^{\prime}
$$

where $\sigma_{i}\left(E^{\prime}\right)$ is the energy-dependent excitation cross section and $E_{i}^{\text {th }}$ is the threshold energy for the excitation in state $i . J_{i}$ in the expression (7) is only due to primary electrons.

The contribution of the secondary electrons to the production is computed separately and is given in the following section.

\section{Excitation by Secondary Electrons}

The ionization of $\mathrm{H}_{2}$ and $\mathrm{H}$ by primary electrons produce secondary electrons which, in turn, can create tertiary electrons. These secondaries and tertiaries also collide with $\mathrm{H}_{2}$ and consequently excite the rotational, vibrational, and electronic states. The secondary electron excitation can be studied by different approaches. We have followed the method of Rees et al. [1969] to compute the secondary electron flux. The production rate of secondary (and higher order) electrons with energy $E_{s}$ is

$$
\eta\left(E_{s}, z\right)=f\left[q\left(\mathrm{H}_{2}^{+}\right)+q\left(H^{+}\right)\right]
$$

where $q\left(\mathrm{H}_{2}^{+}\right)$and $q\left(\mathrm{H}^{+}\right)$are the ionization rate of $\mathrm{H}_{2}$ and $\mathrm{H}$ at altitude $z$ and $f$ is a shape parameter [Opal et al., 1971]. The secondary electron flux at altitude $z$ can be computed by using the following expression:

$$
\phi\left(E_{s}, z\right)=\frac{\int_{E_{s}}^{\infty} \eta(E, z) d E}{[d E / d x]_{e-\mathrm{H}_{2}}+[d E / d x]_{e-\mathrm{H}}+[d E / d x]_{e-e}}
$$

where $[d E / d x]_{e-\mathrm{H}_{2}},[d E / d x]_{e-\mathrm{H}}$, and $[d E / d x]_{e-e}$ are the stopping powers of electrons by $\mathrm{H}_{2}, \mathrm{H}$, and thermal electrons, respectively. We use

$$
\left[\frac{d E}{d x}\right]_{e-e}=\frac{3.37 \times 10^{-12} n(e)^{0.97}}{E_{s}^{0.94}}
$$

[Swartz et al., 1971]. Assuming that the secondary electrons are degraded locally, the volume excitation rate of any state $i$ at altitude $z$ is computed as follows:

$$
P_{i}(z)=n(z) f_{j} \int_{E_{t}^{\text {th }}}^{\infty} \phi\left(E_{s}, z\right) \sigma_{i}\left(E_{s}\right) d E_{s}
$$

The total excitation rate is the sum of the contribution from primary (equation (6)) and secondary (equation (8)) electrons.

\section{Optical Emissions}

The principal ultraviolet emission features observed in the Jovian and Saturnian aurorae are the Lyman $\alpha(1216 \AA)$ line and the Lyman $\left(B^{1} \Sigma_{u}{ }^{+} \rightarrow X^{1} \Sigma_{g}\right)$ and Werner $\left(C^{1} \Pi_{u} \rightarrow{ }^{1} \Sigma_{g}\right)$ bands. The excitation cross sections for the $B$ and $C$ singlets and for the triplet states given in an analytical form by Garvey et al. [1977] are adopted. The values for the singlet states are based on the available laboratory measurements as reviewed by Gerhart [1975], normalized to match their calculated values at $350 \mathrm{eV}$.

According to Heaps et al. [1973], cascading to the $C^{1} \Pi_{u}$ state from the upper singlet states can only increase the population by less than $10 \%$ and has consequently been neglected. However, cascading from the $E, F$ states to the $B$ state is important and must be taken into account. The cross sections for the $E, F$ states are given by Miles et al. [1972]. Collisional deactivation of these emissions can also be neglected, since radiation is faster than quenching. The $B$ state partly radiates into the vibrational continuum of the ground state, giving rise to continuous emissions between 1400 and $1700 \AA$. The intensity $I_{v^{\prime}, v^{\prime \prime}}{ }^{w}$ of a given $\left(v^{\prime}, v^{\prime \prime}\right)$ band in the Werner system can be computed as follows:



The Franck-Condon factors, $q_{v^{\prime}, 0}{ }^{X \rightarrow C}$ and the transition probabilities $A_{v^{\prime} v^{\prime \prime}} c \rightarrow C$ for the $C$ state are taken from the work of Spindler [1969a] and Allison and Dalgarno [1970]. $I_{C}$ is the total intensity of the $C$ state. The intensity $I_{v^{\prime}, v^{\prime}}{ }^{L}$ of a given $\left(v^{\prime}, v^{\prime \prime}\right)$ band in the Lyman system is given by

$$
\begin{array}{r}
I_{v^{\prime} v^{\prime \prime}} L=\left[I_{B} q_{v^{\prime}, 0}^{X \rightarrow B}+I_{E, F} q_{v, 0}^{X \rightarrow E, F} \sum_{v} \frac{A_{v v^{\prime}}, F \rightarrow B}{\sum_{v^{\prime \prime}} A_{v v^{\prime}}}\right] \\
\cdot \frac{A_{v^{\prime} v^{\prime \prime} \rightarrow X}}{\sum_{v^{\prime \prime}}\left(A_{v^{\prime} v^{\prime \prime}} B^{\prime \prime X}+D_{\left.v^{\prime} v^{\prime}\right)}\right)}
\end{array}
$$

where $q_{v^{\prime}, 0}^{X \rightarrow B}$ and $q_{v, 0}{ }^{X-E, F}$ are the Franck-Condon factors for the $B$ and $E, F$ states, respectively. $A_{v^{\prime} v^{\prime}{ }^{B \rightarrow X}}$ is the transition probability for a band from the $B$ to the ground state, $A_{v v^{\prime}}{ }^{E, F \rightarrow B}$ is the transition probability for a band from $E, F$ to the $B$ state. $I_{B}$ and $I_{E, F}$ are the intensities of the $B$ and $E, F$ states, respectively. The second term in the bracket gives the contribution of cascading from the $E, F$ states, 




Fig. 1. Model atmosphere for the Jovian thermosphere.

which contributes $25 \%$ to the total $B$ state population. The Franck-Condon factors for the $B$ state have been calculated by Allison and Dalgarno [1970] as well as the dissociation probability of a particular band $D_{v^{\prime} v^{\prime \prime}}$. For the $E, F$ state, the transition probabilities are computed by using a $q_{v^{\prime} v^{\prime \prime}} v^{3}$ dependence.

The production of Lyman $\alpha(n=2 \rightarrow 1), \mathrm{H} \alpha(n=3 \rightarrow 2)$ and $\mathrm{H} \beta(n=4 \rightarrow 2)$ emissions can result from the dissociative reaction of $\mathrm{H}_{2}$. Vroom and De Heer [1969] have measured the absolute cross sections for the production of $\mathrm{H} \alpha$ and $\mathrm{H} \beta$ emissions between $50 \mathrm{eV}$ and $6 \mathrm{keV}$. Below 50 $\mathrm{eV}$, the measurements of Weaver and Hughes [1970] are adopted. Cascading effects in Balmer emissions are negligible. Below $340 \mathrm{eV}$, the Lyman $\alpha$ cross section from Mumma and Zipf [1971] is used. Above this energy, the measurements of Vroom and De Heer [1969] have been adopted with the correction of polarization effects suggested by Mumma and Zipf. The excitation cross sections of the $n=1-6$ states of atomic hydrogen by electron impact have been given by Olivero et al. [1973] and were used to calculate the $\mathrm{Ly} \alpha, \mathrm{H} \alpha$ and $\mathrm{H} \beta$ excitation rates.

\section{The Jovian Aurora}

Observations have shown that the Jovian mesosphere is isothermal at $170 \mathrm{~K}$ [Hunten, 1976]. However, the temperature profile above the mesopause exhibits large variations. Atreya and Donahue [1976] have modeled the Pioneer 10 and 11 electron density by assuming an exospheric temperature of $1050 \mathrm{~K}$, but Fjeldbo et al. [1976] deduced a value of $850 \mathrm{~K}$ from radio occultation measurements. However, various measurements made with Voyager showed that the temperature of the exosphere had increased to above $1000 \mathrm{~K}$ in 1979. In addition to the EUV solar radiation, possible heat sources are gravity waves and particle precipitation [Hunten and Dessler, 1977]. In this model we have adopted a value of $1400 \mathrm{~K}$, slightly higher than the measured equatorial value of $1100 \pm 200 \mathrm{~K}$ [Festou et al., 1981] to account for the highlatitude heating described below. We have adopted a temperature profile similar to that derived by Festou et al. [1981] from the stellar occultation measurements of Voyager 1 which is characterized by a lapse rate of $1 \mathrm{~K} / \mathrm{km}$ in the thermosphere. The altitude is measured from where the total density is $5 \times 10^{13} \mathrm{~cm}^{-3}$, which corresponds to the mesopause [Hunten and Dessler, 1977] and to a pressure level of about $1 \mu \mathrm{bar}$. Voyager measurements indicate that the helium content in the Jupiter homosphere is close to $11 \%$. Recent determinations of the eddy diffusion coefficient have estimated that $\mathrm{K}$ lies between 1 and $3 \times 10^{6} \mathrm{~cm}^{2} \mathrm{~s}^{-1}$ near homopause [McConnell et al., 1981], where the total density is about $7 \times 10^{13} \mathrm{~cm}^{-3}$. Above the homopause the fractional abundance of helium becomes rapidly negligible.

There are large uncertainties about the thermospheric atomic hydrogen distribution, which is strongly dependent on the eddy diffusion and source function profiles. Yung and Strobel's [1980] densities of $\mathrm{H}$ vary by 2 orders of magnitude, depending on the set of parameters adopted. If a mixing ratio of $8.5 \times 10^{-3}$ is adopted at the altitude where the total density is $1 \times 10^{12} \mathrm{~cm}^{-3}$ in agreement with their $\mathrm{C}_{12}$ model, $\mathrm{H}$ becomes important only above $800 \mathrm{~km}$ (case $\mathrm{A}$ ). To investigate the effects of a lower atomic hydrogen abundance, calculations have also been made with an $\mathrm{H}$ density increased by an order of magnitude (case B). The model temperature and density profiles are shown in Figure 1. In the absence of direct measurement of the electron flux in the auroral zones, it is necessary to adopt an energy spectrum. We have used a Maxwellian distribution outside the atmosphere, since this form is usually a good approximation to the energy spectrum of electrons measured in the earth's aurora:

$$
\Phi\left(E_{0}\right)=F_{0} \frac{E_{0}}{\alpha^{2}} \exp \left(-\frac{E_{0}}{\alpha}\right) \mathrm{el} \mathrm{cm}^{-2} \mathrm{~s}^{-1} \mathrm{eV}^{-1}
$$

where $F_{0}$ is the total particle flux and $\alpha$ determines the hardness of the spectrum. For illustration purposes, we have adopted values of $\alpha$ of $0.1,0.4,2$, and $10 \mathrm{keV}$. In these model calculations, the total energy flux is $1 \mathrm{erg} \mathrm{cm}^{-2} \mathrm{~s}^{-1}$. Figure 2 shows the $\mathrm{H}_{2}{ }^{+}$and $\mathrm{H}^{+}$production rates for these values of $\alpha$. As mentioned before, the IUE auroral spectrum sets an upper limit of $\sim 2 \times 10^{17} \mathrm{CH}_{4}$ molecules $/ \mathrm{cm}^{2}$ above the auroral emission peak for an emission angle of $\sim 60^{\circ}$. This number can be compared with the $\mathrm{CH}_{4}$ vertical column density of $2.5-5 \times 10^{17} \mathrm{~cm}^{-2}$ present above our reference level if the methane mixing ratio of $1.8 \times 10^{-3}$ determined by Sato and Hansen [1979] in the homosphere is adopted. Consequently, observations indicate the absence of hydrocarbon absorption in the spectrum and locate the $\mathrm{H}_{2}$ ionization and emission maximum above, or close to, the reference level of $5 \times 10^{13} \mathrm{~cm}^{-3}$. This altitude corresponds to an upper limit of $\alpha$ of the order of $10 \mathrm{keV}$, i.e., to a mean electron energy of $20 \mathrm{keV}$.

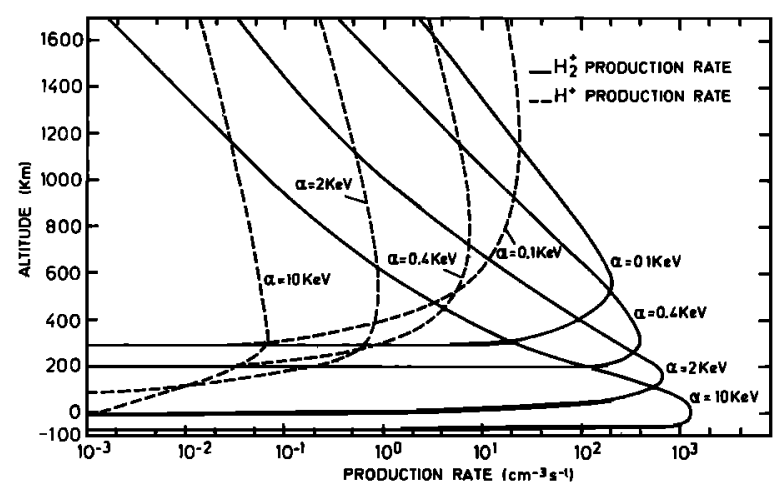

Fig. 2. Altitude distribution of the production rates in the Jovian auroral zones due to an isotropic auroral electron precipitation with a Maxwellian energy distribution and model atmosphere from case A. 


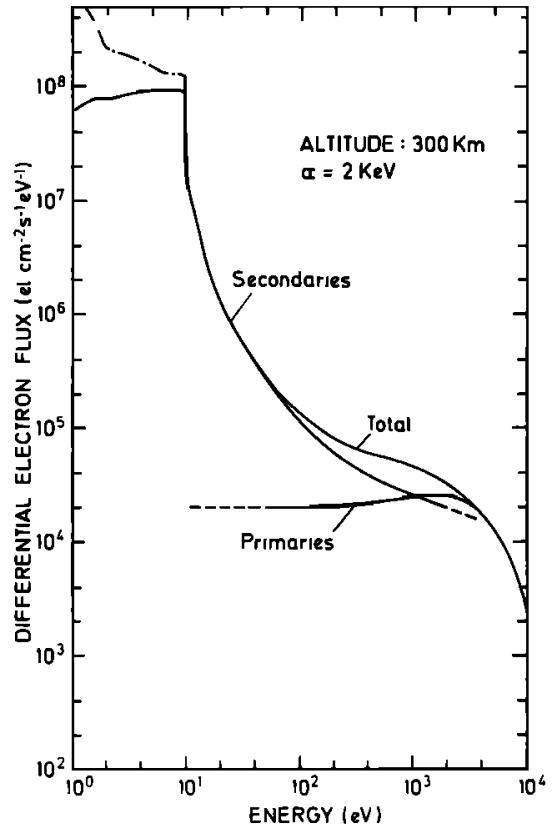

Fig. 3. Calculated electron energy spectrum near the ionization maximum (case A). The dashed-dotted curve shows the energy distribution without collisions with thermal electrons.

The secondary and degraded primary electron fluxes at $300 \mathrm{~km}$ are shown in Figure 3 as well as the total flux. Only in a transition region between $100 \mathrm{eV}$ and $2 \mathrm{keV}$ are secondaries and primaries both important. Below $100 \mathrm{eV}$ (dotted curve) the calculated degraded primary distribution is nearly constant and cannot be evaluated accurately in the continuous slowing down approximation. This is a consequence of the fact that only a narrow range of the initial distribution adjacent to the cutoff energy $E_{0}$ contributes to the local lowenergy tail of the degraded primaries. Actually, fluctuations of the range of the individual electrons will modify the distribution, but only Monte Carlo methods can model this effect quantitatively. Thermal electron heating plays an important role below about $10 \mathrm{eV}$ as illustrated in Figure 2. The electron density profile is calculated from the photochemical equilibrium for $\mathrm{H}^{+}, \mathrm{H}_{2}{ }^{+}, \mathrm{H}_{3}{ }^{+}$, and electrons. The direct production rate of $\mathrm{H}_{2}{ }^{+}$and $\mathrm{H}^{+}$by electron impact on $\mathrm{H}_{2}$ and $\mathrm{H}$ are given by (1). The fraction of dissociative ionization is $6 \%$ of the total $\mathrm{H}_{2}$ ionization. The list of chemical reactions is given in Table 2 . Two paths are energetically possible for reaction (3), but the branching ratio is unknown and only the total recombination coefficient has been measured. The loss rate of $\mathrm{H}^{+}$is enhanced by collision with vibrationally excited $\mathrm{H}_{2}$ as suggested by Atreya et al. [1979]. As shown in Figure 4, $\mathrm{H}^{+}$is the major

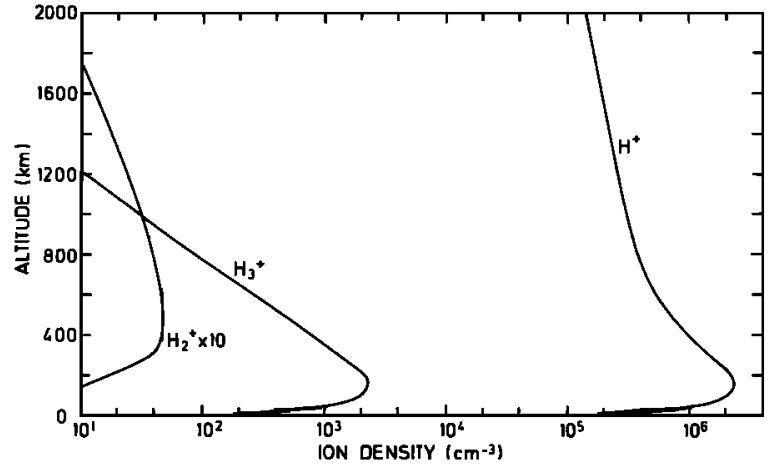

Fig. 4. Ion density distribution calculated for a $1 \mathrm{erg} \mathrm{cm}^{-2} \mathrm{~s}^{-1}$ electron precipitation with $\alpha=2 \mathrm{keV}$ and model atmosphere from case $A$.

ion in the auroral thermosphere and $\mathrm{H}_{3}{ }^{+}$, the terminal ion, dominates over $\mathrm{H}_{2}{ }^{+}$. The peak electron density for a 1 erg $\mathrm{cm}^{-2} \mathrm{~s}^{-1}$ precipitation exceeds $1 \times 10^{6} \mathrm{~cm}^{-3}$ and increases linearly with the ionization rate if reaction (2) is the main loss process for $\mathrm{H}^{+}$. Figure 5 illustrates the total electron flux at infinity and different altitudes located above, at and below the ionization maximum.

The volume excitation rate of $B^{1} \Sigma_{u}{ }^{+}, C^{1} \Sigma_{u}$ of $\mathrm{H}_{2}$, and $n=$ 2, 3, 4 states of atomic hydrogen are shown in Figure 6. For the $C$ state, $\mathrm{H} \alpha$ and $\mathrm{H} \beta$, the volume excitation rates are equal to volume emission rates, since these states radiate completely. Consequently, the apparent intensity can be obtained by integrating the volume excitation rate over the vertical column. Some of the transitions from the $B^{1} \Sigma_{u}{ }^{+}$ state lead to the dissociation of $\mathrm{H}_{\hat{2}}$. The fraction of dissociation is computed by using the Franck-Condon factors [Spindler, 1969b] and the transition probabilities [Allison and Dalgarno, 1970] used in formula (10). It is found that about $30 \%$ of the $B$ state population dissociates $\mathrm{H}_{2}$ and give rise to continuum emission in the $1400-1700 \AA$ range. This contribution is taken into account when comparing these model calculations with the Voyager $\mathrm{H}_{2}$ EUV emission.

Column production rates for the Werner and Lyman bands, $\mathrm{H} \alpha, \mathrm{H} \beta$ are listed in Table 3 for various values of $\alpha$ and for atomic hydrogen models A and B. The Ly $\alpha$ line optical thickness is very large and the radiative transfer problem is not considered here. Consequently, the column production rate of $L y \alpha$ cannot be compared to the emission rates. As $\alpha$ decreases, the altitude of the excitation maximum moves upward into a region richer in atomic hydrogen, and the relative brightness of the EUV $\mathrm{H}_{2}$ emissions decreases, since the $\mathrm{H} / \mathrm{H}_{2}$ abundance ratio increases. Changing from model A to model B has a similar effect on the intensity ratio. The Voyager measurements of these emissions in the Jovian aurora indicate a total $\mathrm{H}_{2}$ intensity of about $80 \mathrm{kR}$

TABLE 2. Reactions Considered in Jovian Auroral Ionosphere

\begin{tabular}{|c|c|c|c|c|}
\hline $\begin{array}{l}\text { Reaction } \\
\text { Number }\end{array}$ & Reaction & $\begin{array}{c}\text { Exothermicity, } \\
\mathrm{eV}\end{array}$ & Rate Coefficient & Reference \\
\hline 1 & $\mathrm{H}_{2}^{+}+\mathrm{H}_{2} \rightarrow \mathrm{H}_{3}^{+}+\mathrm{H}$ & 1.6 & $2.1 \times 10^{-9} \mathrm{~cm}^{3} \mathrm{~s}^{-1}$ & $\begin{array}{l}\text { Theard and Huntress } \\
\text { [1974] }\end{array}$ \\
\hline $\begin{array}{l}2 \\
3\end{array}$ & $\begin{aligned} \mathbf{H}^{+}+\mathbf{H}_{2}(v) & \rightarrow \mathbf{H}_{2}^{+}+\mathbf{H} \\
\mathbf{H}_{3}^{+}+e & \rightarrow \mathbf{H}_{2}+\mathbf{H} \\
& \rightarrow \mathrm{H}+\mathrm{H}+\mathrm{H}\end{aligned}$ & $\begin{array}{l}-1.8(v=0) \\
9.3 \\
4.8\end{array}$ & $\begin{array}{l}4.3 \times 10^{-16} \mathrm{~cm}^{3} \mathrm{~s}^{-1} \\
4.8 \times 10^{-7} \mathrm{~cm}^{3} \mathrm{~s}^{-1}\end{array}$ & $\begin{array}{l}\text { Atreya et al. }[1979] \\
\text { Leu et al. }[1973]\end{array}$ \\
\hline $\begin{array}{l}4 \\
5\end{array}$ & $\begin{array}{l}\mathrm{H}_{2}^{+}+e \rightarrow \mathrm{H}+\mathrm{H} \\
\mathrm{H}^{+}+e \rightarrow \mathrm{H}+h v\end{array}$ & $\begin{array}{l}10.9 \\
13.6\end{array}$ & $\begin{array}{l}1.0 \times 10^{-8} \mathrm{~cm}^{3} \mathrm{~s}^{-1} \\
6.6 \times 10^{-12} \mathrm{~cm}^{3} \mathrm{~s}^{-1}\end{array}$ & $\begin{array}{l}\text { Hunten [1969] } \\
\text { Bates and Dalgarno } \\
\text { [1962] }\end{array}$ \\
\hline
\end{tabular}






Fig. 5. Total electron energy spectra at different altitudes for an incident Maxwellian distribution and model atmosphere from case A.

This value implies that an energy flux of the order of 8-10 ergs $\mathrm{cm}^{-2} \mathrm{~s}^{-1}$ was precipitated into the Jovian auroral zones at the time of the Voyager 2 encounter if the precipitation was hard ( $\alpha \geq 2 \mathrm{keV}$ ) or if the $\mathrm{H}$ abundance was below model $A$. In the case of a soft precipitation or a thermosphere rich in atomic hydrogen, the incoming electron flux must be revised upward according to the results listed in Table 3.

The efficiency for the excitation of the total $\mathrm{H}_{2}$ EUV emission is $10.6 \mathrm{kR} / \mathrm{erg} \mathrm{cm}^{-2} \mathrm{~s}^{-1}$ in this calculation for a pure $\mathrm{H}_{2}$ atmosphere. This value is obtained by using Garvey et al.'s cross sections yielding effective values of $3.6 \times 10^{-17}$ $\mathrm{cm}^{-2}$ and $3.0 \times 10^{-17} \mathrm{~cm}^{-2}$ at $100 \mathrm{eV}$ for the $B$ and $C$ states, respectively. If these values are revised in the future, the $\mathrm{H}_{2}$ emission efficiency should be modified accordingly.

\section{Heating Efficiency and Dissociation}

The kinetic energy of the incident electrons can be converted to thermal energy by several mechanisms. The lowest excited electronic state of $\mathrm{H}_{2}$ is $b^{3} \Sigma_{u}{ }^{+}$with a threshold of 10 eV. Electrons below $10 \mathrm{eV}$ can lose their energy to the ambient gas, through elastic collisions with $\mathrm{H}_{2}$ or by exciting the rotational and vibrational states of $\mathrm{H}_{2}$. These states have very long radiation lifetimes, and therefore these states are collisionally deactivated. Ultimately, the energy of electrons below $10 \mathrm{eV}$ is deposited locally as thermal energy. All the higher lying triplet states essentially decay to the $b^{3} \Sigma_{u}{ }^{+}$ state. This state dissociates into two hydrogen atoms with a mean kinetic energy of $5.5 \mathrm{eV}$ [Cravens et al., 1975]. Dissociation through the $\mathrm{B}^{1} \Sigma_{u}{ }^{+}$state can also be a source of neutral heating. Cascading from higher lying $E, F^{1} \Sigma_{g}{ }^{+}$states increases the population of the $B^{1} \Sigma_{u}{ }^{+}$state and modifies its vibrational distribution. Heaps [1976] has adopted an average energy between 0.05 and $0.15 \mathrm{eV}$ per excitation, which is converted into heat by radiative dissociation. Dissociative excitation of $\mathrm{H}_{2}$ by primary and secondary electrons yields $4.87 \mathrm{eV}$ kinetic energy for the fragments for $n=2$ [Cravens et al., 1975]. The dissociative recombination of $\mathrm{H}_{2}{ }^{+}$and $\mathrm{H}_{3}{ }^{+}$ ions with ambient electrons is a major source of neutral heating because of the high ionization rate. The $\mathrm{H}_{3}{ }^{+}+e$ recombination is very fast and yields 9.3 or $4.8 \mathrm{eV}$ to the fragments (Table 2). Table 4 gives the heating efficiency in the Jovian aurora for $\alpha=2 \mathrm{keV}$ and three different model atmospheres. Dissociative recombination of ions with thermal electrons is the major source of neutral heating in the case of a $\mathrm{H}_{2}$-dominated atmosphere. This reaction contributes by $27 \%$ to the heating efficiency. The second major source is the cooling of electrons below $10 \mathrm{eV}$. The total heating efficiency is 0.43 , a value quite close to the $40 \%$ calculated by Heaps [1976] and in good agreement with the study of Waite et al. [1982]. By using the total energy flux deduced in the previous section, we estimate the particle heat production to about $4 \mathrm{ergs} / \mathrm{cm}^{2} \mathrm{~s}$ in the auroral zone or $10^{20} \mathrm{ergs} / \mathrm{s}$ for the entire planet in the case of an $\mathrm{H}_{2}$ atmosphere or hard precipitation.

Molecular hydrogen will be partly dissociated under the effect of electron bombardment. The atomic hydrogen production can be evaluated considering that $37 \mathrm{eV}$ are required to create an electron-ion pair and that two $\mathrm{H}$ atoms are ultimately produced per $\mathrm{H}_{2}$ ionization if $\mathrm{H}_{3}{ }^{+}$recombination gives $\mathrm{H}_{2}+\mathrm{H}$. Consequently, the 10 ergs $\mathrm{cm}^{-2} \mathrm{~s}^{-1}$ energy flux deduced previously implies a production rate of about $3.5 \times 10^{11} \mathrm{H}$ atoms cm $\mathrm{cm}^{-2} \mathrm{~s}^{-1}$ in the auroral zones. Averaged over the whole planet, the mean column production rate is $\sim 1 \times 10^{10} \mathrm{H}$ atoms $\mathrm{cm}^{-2} \mathrm{~s}^{-1}$. This value is within the range of flux values required by Yung and Strobel [1980] from their analysis of the Ly $\alpha$ emission measured by the Voyager UVS and exceeds the solar EUV production by at least 1 order of magnitude.

\section{The Saturnian Aurora}

The model described before is directly applicable to the Saturn aurora. The 2-15 $\mathrm{kR} \mathrm{H}_{2}$ emission observed during the Voyager 1 encounter corresponds to energy fluxes varying between 0.2 and $1.5 \mathrm{erg} \mathrm{cm}^{-2} \mathrm{~s}^{-1}$ and a local heating rate of $0.04-0.3 \mathrm{erg} \mathrm{cm}^{-2} \mathrm{~s}^{-1}$. The latter value is much larger than the solar EUV energy input of about $4 \times 10^{-3} \mathrm{erg} \mathrm{cm}^{-2} \mathrm{~s}^{-1}$. The local atomic hydrogen production rate is thus $0.7-5 \times$ $10^{10} \mathrm{H}$ atoms $\mathrm{cm}^{-2} \mathrm{~s}^{-1}$. The overall heating rate from the auroral precipitation is $1-7 \times 10^{17}$ ergs $\mathrm{cm}^{-2} \mathrm{~s}^{-1}$, and the globally averaged hydrogen production is $0.9-7 \times 10^{7}$ atoms $\mathrm{cm}^{-2} \mathrm{~s}^{-1}$.

The altitude distribution of the ionization and hydrogen emissions have been calculated by using this model. The

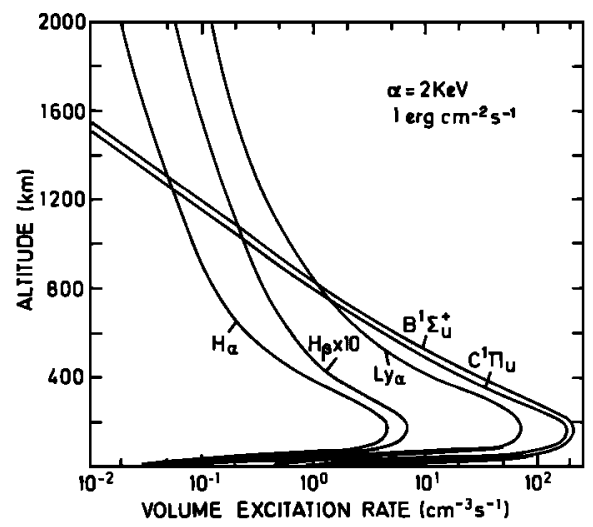

Fig. 6. Volume excitation rate of the $\mathrm{H}_{2} B$ and $C$ states and three lines of atomic hydrogen in the Jovian aurora for model atmosphere with case $A$. 
TABLE 3. Column Production Rates in Jovian Aurora

\begin{tabular}{|c|c|c|c|c|c|c|c|}
\hline \multirow[b]{3}{*}{ Emission } & \multicolumn{7}{|c|}{ Column Production Rates, $\mathbf{k R}$} \\
\hline & \multicolumn{2}{|c|}{$\alpha=0.1 \mathrm{keV}$} & \multicolumn{2}{|c|}{$\alpha=0.4 \mathrm{keV}$} & \multicolumn{2}{|c|}{$\alpha=2.0 \mathrm{keV}$} & \multirow{2}{*}{$\frac{\alpha=2.0 \mathrm{keV}}{\text { Pure } \mathrm{H}_{2}}$} \\
\hline & Case $\mathbf{A}$ & Case B & Case $\mathbf{A}$ & Case B & Case A & Case B & \\
\hline $\begin{array}{l}\text { Lyman bands } \\
\text { Werner bands } \\
\text { H } \alpha \\
\text { H } \beta\end{array}$ & $\begin{array}{c}3.2 \mathrm{KR} \\
3.0 \mathrm{KR} \\
660 \mathrm{R} \\
50 \mathrm{R}\end{array}$ & $\begin{aligned} 112 \mathrm{R} \\
95 \mathrm{R} \\
4.6 \mathrm{KR} \\
260 \mathrm{R}\end{aligned}$ & $\begin{aligned} 1.4 \mathrm{KR} \\
4.2 \mathrm{KR} \\
280 \mathrm{R} \\
28 \mathrm{R}\end{aligned}$ & $\begin{array}{l}845 \mathrm{R} \\
750 \mathrm{R} \\
2.5 \mathrm{KR} \\
147 \mathrm{R}\end{array}$ & $\begin{array}{c}4.9 \mathrm{KR} \\
4.6 \mathrm{KR} \\
125 \mathrm{R} \\
20 \mathrm{R}\end{array}$ & $\begin{array}{c}3.1 \mathrm{KR} \\
2.8 \mathrm{KR} \\
540 \mathrm{R} \\
36 \mathrm{R}\end{array}$ & $\begin{array}{c}5.4 \mathrm{KR} \\
5.2 \mathrm{KR} \\
125 \mathrm{R} \\
20 \mathrm{R}\end{array}$ \\
\hline
\end{tabular}

atmosphere has an exospheric temperature of $850 \mathrm{~K}$, in agreement with the Voyager observations. The vertical structure adopted is similar to the Jupiter thermosphere with a reference level at $n\left(\mathrm{H}_{2}\right)=5 \times 10^{13} \mathrm{~cm}^{3}$ and a temperature of $150 \mathrm{~K}$ at this altitude. In the case of $\alpha=2 \mathrm{keV}$, the peak of ionization is located at that altitude where the $\mathrm{H}_{2}$ density is $6.5 \times 10^{10} \mathrm{~cm}^{-3}$.

\section{ConClusions}

The model described in this paper provides a useful tool to calculate vertical distributions of hydrogen emissions, ionization, local electron energy fluxes, ion composition, particle heating rates, and dissociation rates. The present main limitations are (1) the total lack of knowledge of the energy spectrum of the precipitated particles, (2) the exact thermal structure of the planets' thermospheres, and (3) the abundance and distribution of thermospheric atomic hydrogen. Nevertheless, a few important conclusions can be drawn from the comparison between the Voyager EUV observation and these model calculations. This comparison indicates that, for Jupiter, high-latitude particle precipitation plays an important role as a source of heat and dissociation. For example, we predict a local heating rate of about $4 \mathrm{ergs}^{-2}$ $\mathrm{s}^{-1}$, exceeding by far the ionizing solar flux of $0.01 \mathrm{erg} \mathrm{cm}^{-2}$ $\mathrm{s}^{-1}$. Spectroscopic evidence indicates that all or most of the energy is deposited by electrons in the thermosphere. Consequently, both the magnitude and the altitude of the particle and possibly Joule heating are adequate to maintain a high exospheric temperature. Similarly, the model predicts a globally averaged production of hydrogen atoms of the order of $1 \times 10^{10} \mathrm{~cm}^{-2} \mathrm{~s}^{-1}$, which dominates the photochemical sources, in agreement with the analysis of the Ly $\alpha$ radiation field. The absence of detectable $\mathrm{H}_{2}$ emission on the Jupiter mid-latitude nightside implies that these strong sources of heat and dissociation are confined to a limited portion of the planet. Horizontal and vertical transport will redistribute heat and atoms. A global circulation may be driven as a consequence of the large heat input gradients. Temporal and spatial variations in the precipitated electron flux will induce

TABLE 4. Heating Efficiency, $\alpha=2 \mathrm{keV}$

\begin{tabular}{lccc}
\hline & \multicolumn{3}{c}{ Model Atmosphere } \\
\cline { 2 - 4 } & $\begin{array}{c}\text { Pure } \mathrm{H}_{2} \\
\text { Atmosphere }\end{array}$ & Case A & Case B \\
\hline Dissociative recombination & 0.27 & 0.24 & 0.15 \\
Triplet states & 0.04 & 0.04 & 0.02 \\
Direct dissociation of $\mathrm{H}_{2}$ & 0.04 & 0.04 & 0.02 \\
Low-energy electrons & & & \\
$\begin{array}{c}\text { (<10 eV) } \\
\text { Singlet states } \\
\text { Elastic scattering }\end{array}$ & 0.06 & 0.06 & 0.06 \\
Total & 0.01 & 0.01 & 0.005 \\
\hline
\end{tabular}

a strong but probably time dependent source of atomic hydrogen. It is likely that this can at least partly account for the variability observed by Clarke et al. [1980] in the Ly $\alpha /$ $\mathrm{H}_{2}$ bands intensity ratio. Other possible sources of variations of this ratio include (1) geometrical effect of the wide field of view of the IUE instrument coupled with different spatial extent of the $\mathrm{H}_{2}$ and $\mathrm{Ly} \alpha$ sources; (2) temperature variations affecting the $\mathrm{H}_{2}$ spectral distribution; (3) proton excitation of the hydrogen emission; and (4) absorption by hydrocarbons in the case of hard precipitation. Combination of these factors could easily account for the observed variability, but more observations are needed before the importance of these effects can be evaluated quantitatively. In any case, the heat budget of the upper Jovian atmosphere will be affected by the particle precipitation.

Since dissociative recombination of $\mathrm{H}_{3}{ }^{+}$is the main source of $\mathrm{H}$ atoms, an excess energy of about $6.2 \mathrm{or} 1.6 \mathrm{eV}$ will be carried as kinetic energy of the fragments, depending on the path followed in the recombination process. Although their velocity remains smaller than the escape velocity (19.4 $\mathrm{eV}$ for $\mathbf{H}$ atoms on Jupiter), a fraction of the hot atoms will reach the exosphere and form a hot atom corona surrounding the polar regions.

Although less dramatic heating and dissociation rates are predicted for Saturn, the effects of precipitation may be quite important in the vicinity of the auroral zones. Substantial increases in the electron and ion densities are also predicted in the high-latitude regions of both planets.

We note, finally, that the thermospheric thermal structure of Jupiter may be entirely controlled in the polar regions by the particle heat input and may exhibit important latitudinal and temporal variations. Consequently, models derived from mid-latitude occultation experiments may be inconsistent with the local inputs. Local measurements of the thermospheric composition and structure are needed over long periods of time. These observations, coupled with twodimensional or three-dimensional modeling of the transport of heat and atoms, will help understanding the global budget of the planet.

Acknowledgments. One of the authors (J. C. G.) is supported by the Belgian Foundation for Scientific Research (FNRS). V. Singh acknowledges the University of Liège for a research fellowship. The authors also thank T. E. Cravens and S. Durrance for valuable discussions.

The Editor thanks Y. L. Yung and D. E. Shemansky for their assistance in evaluating this paper.

\section{REFERENCES}

Allison, A. C., and A. Dalgarno, Band oscillator strengths and transition probabilities for the Lyman and Werner band systems of $\mathrm{H}_{2}, \mathrm{HD}$ and $\mathrm{D}_{2}$, At. Data, $1,289,1970$.

Atreya, S. K., and T. M. Donahue, Model ionosphere of Jupiter, in Jupiter, edited by T. Gehrels, pp. 304-318, University of Arizona Press, Tucson, 1976. 
Atreya, S. K., Y. L. Yung, T. M. Donahue, and E. S. Barker, Search for Jovian auroral hot spots, Astrophys. J., 218, L83-L87, 1977.

Atreya, S. K., T. M. Donahue, and J. H. Waite, An interpretation of the Voyager measurement of Jovian electron density profiles, Nature, 280, 795-796, 1979.

Bates, D. R., and A. Dalgarno, Electronic Recombination, Atomic and Molecular Processes, edited by D. R. Bates, Academic, New York, 1962.

Broadfoot, A. L., M. J. S. Belton, P. Z. Takacs, B. R. Sandel, D. E. Shemansky, J. B. Holberg, A. M. Ajello, S. K. Atreya, T. M. Donahue, J. W. Moos, J. L. Bertaux, J. E. Blamont, D. F. Strobel, J. C. McConnell, A. Dalgarno, R. Goody, and M. B. McElroy, Extreme ultraviolet observations from Voyager 1 encounter with Jupiter, Science, 204, 979-982, 1979.

Broadfoot, A. L., B. R. Sandel, D. E. Shemansky, J. C. McConnell, G. R. Smith, J. B. Holberg, S. K. Atreya, T. M. Donahue, D. F. Strobel, and J. L. Bertaux, Overview of the Voyager ultraviolet spectroscopy results through Jupiter encounter, J. Geophys. Res., 86, 8259-8284, 1981a.

Broadfoot, A. L., B. R. Sandel, D. E. Shemansky, J. B. Holberg, G. R. Smith, D. F. Strobel, J. C. McConnell, S. Kumar, D. M. Hunten, S. K. Atreya, T. M. Donahue, H. W. Moos, J. L. Bertaux, J. E. Blamont, R. B. Pomphrey, and S. Linick, Extreme ultraviolet observations from Voyager 1 encounter with Saturn, Science, 212, 206-211, 1981b.

Clarke, J. T., H. W. Moos, S. K. Atreya, and A. L. Lane, Observations from earth orbit and variability of the polar aurora on Jupiter, Astrophys. J., 241, 179-182, 1980.

Clarke, J. T., H. W. Moos, S. K. Atreya, and A. L. Lane, IUE detection of bursts of $\mathrm{H}$ Ly $\alpha$ emission from Saturn, Nature, 290 , 226-227, 1981.

Cook, A. F., A. Vallance Jones, and D. E. Shemansky, Visible auroral in Jupiter atmosphere?, J. Geophys. Res., 86, 8793-8796, 1981.

Cravens, T. E., G. A. Victor, and A. Dalgamo, The absorption of energetic electrons by molecular Hydrogen gas, Planet. Space Sci., 23, 1059-1070, 1975 .

Dulk, G. A., J. A. Eddy, and J. P. Emerson, Search for visual auroral on Jupiter, Astrophys. J., 159, 1123-1124, 1970.

Durrance, S. T., P. D. Feldman, H. W. Moos, The spectrum of the Jovian aurora 1100-1700 A, Geophys. Res. Lett., in press, 1982.

Edgar, B. C., H. S. Porter, and A. E. S. Green, Proton energy deposition in molecular and atomic oxygen and applications to the polar cap, Planet. Space Sci., 23, 787-804, 1975.

Festou, M. C., S. K. Atreya, T. M. Donahue, B. R. Sandel, D. E. Shemansky, and A. L. Broadfoot, Composition and temperature profiles of the Jovian upper atmosphere determined by the Voyager ultraviolet stellar occultation experiment, J. Geophys. Res., 86, 5715-5725, 1981.

Fjeldbo, G., A. Kliore, B. Seidel, D. Sweetnam, and P. Woiceshyn, The Pioneer 11 radio occultation measurements of the Jovian ionosphere, in Jupiter, edited by $\mathrm{T}$. Gehrels, University of Arizona Press, Tucson, Ariz., 1976.

Garvey, R. H., H. S. Porter, and A. E. S. Green, Relativistic yield spectra for $\mathrm{H}_{2}, J$. Appl. Phys., 48, 4353-4359, 1977.

Gerhart, D. E., Comprehensive optical and collision data for radiation action, I, $\mathrm{H}_{2}, J$. Chem. Phys., 62, 821-832, 1975.

Goertz, C. K., Proton aurora on Jupiter's nightside, Geophys. Res. Lett., 7, 365-368, 1980.

Heaps, M. G., J. N. Bass, and A. E. S. Green, Electron excitation of a Jovian aurora, Icarus, 20, 297-303, 1973.

Heaps, M. G., The roles of particle precipitation and Joule heating in the energy balance of the Jovian thermosphere, Icarus, 29, 273$281,1976$.

Hunten, D. M., The upper atmosphere of Jupiter, J. Atmos. Sci., 26, 826-834, 1969.

Hunten, D. M., Atmosphere and ionospheres, in Jupiter, edited by T. Gehrels, University of Arizona Press, Tucson, Ariz., 1976.

Hunten, D. M., and A. J. Dessler, Soft electrons as a possible heat source for Jupiter's thermosphere, Planet. Space Sci., 25, 817$821,1977$.

Hunter, J. H., $\mathrm{H}_{\alpha}$ auroral activity of Jupiter, Nature, 223, 388-389, 1969.
Judge, D. L., F. M. Wu, and R. W. Carlson, Ultraviolet photometer observations of the Saturnian system, Science, 207, 431-434, 1980

Leu, M. T., M. A. Biondi, and R. Johnsen, Dissociative recombination of electrons with $\mathrm{H}_{3}{ }^{+}$and $\mathrm{H}_{5}{ }^{+}$ion, Phys. Rev., 8, 413-419, 1973.

McConnell, J. C., B. R. Sandel, and A. L. Broadfoot, Airglow from Jupiter's nightside and crescent ultraviolet spectrometer observations from Voyager 2, Icarus, 43, 128-142, 1980.

McConnell, J. C., B. R. Sandel, and A. L. Broadfoot, Voyager UV spectrometer observations of $\mathrm{He} 584 \mathrm{~A}$ dayglow at Jupiter, Planet. Space. Sci., 29, 283-292, 1981.

Miles, W. T., R. Thompson, and A. E. S. Green, Electron impact cross sections and energy deposition in molecular hydrogen, $J$. Appl. Phys., 43, 678-686, 1972.

Mumma, M. J., and E. C. Zipf, Dissociative excitation of vacuum ultraviolet emission features by electron impact on molecular gases, $\mathrm{I}, \mathrm{H}_{2}$ and $\mathrm{O}_{2}$, J. Chem. Phys., 55, 1661-1669, 1971.

Olivero, J. J., J. N. Bass, and A. E. S. Green, Photoelectron excitation of the Jupiter airglow, J. Geophys. Res., 78, 2812-2826, 1973.

Opal, C. B., W. K. Peterson, and E. C. Beatty, Measurements of secondary electron spectra produced by electron impact ionization of a number of simple gases, J. Chem. Phys., 55, 4100-4106, 1971.

Peterson, L. R., and A. E. S. Green, The relation between ionization yields, cross sections, and loss functions, J. Phys., B1, 1131$1140,1968$.

Rees, M. H., A. I. Stewart, and J. C. G. Walker, Secondary electrons in aurora, Planet. Space Sci., 17, 1997-2008, 1969.

Sandel, B. R., D. E. Shemansky, A. L. Broadfoot, J. L. Bertaux, J. E. Blamont, M. J. S. Belton, J. M. Ajello, J. B. Holberg, S. K. Atreya, T. M. Donahue, H. W. Moos, D. F. Strobel, J. C. McConnell, A. Dalgarno, R. Goody, M. B. McElroy, and P. Z. Takacs, Extreme ultraviolet observations from Voyager encounter with Jupiter, Science, 206, 962-966, 1979.

Sato, M., and J. E. Hansen, Jupiter's atmospheric composition and cloud structure deduced from absorption bands in reflected sunlight, J. Atmos. Sci., 36, 1133-1145, 1979.

Schwitters, M. T., A search for Jovian limb aurorae, Icarus, 9, 570$573,1968$.

Spindler, R. J., Jr., Franck-Condon factors for the band systems of molecular hydrogen, 1 , The $\left(B^{1} \Sigma_{u}{ }^{+}-X^{1} \Sigma_{g}{ }^{+}\right),\left(I^{1} \Pi_{g}-B^{1} \Sigma_{u}{ }^{+}\right)$and $\left.d^{3} \Pi_{u}-a^{3} \Sigma_{g}^{+}\right)$systems, J. Quant. Spectrosc. Radiat. Transfer, 9 , $597,1969 a$

Spindler, R. J., Jr., Franck-Condon factors for the band systems of molecular hydrogen, 2 , The $\left(C^{1} \Pi_{u}-X^{1} \Sigma_{g}{ }^{+}\right),\left(D^{1} \Pi_{u}-X^{1} \Sigma_{g}^{+}\right)$and $\left(h^{3} \Sigma_{g}{ }^{+}-c^{3} \Pi_{w}\right)$ systems, J. Quant. Spectrosc. Radiat. Transfer, 9, $627,1969 b$

Swartz, W. E., J. S. Nisbet, and A. E. S. Green, Analytic expression for the energy transfer rate from photoelectron to thermal electrons, J. Geophys. Res., 76, 8425-8426, 1971.

Theard, L. P., and W. T. Huntress, Jr., Ion molecule reactions and vibrational deactivation of $\mathrm{H}_{2}{ }^{+}$ions in mixtures of hydrogen and helium, J. Chem. Phys., 60, 2840-2848, 1974.

Valentine, J. M., and S. C. Curran, Average energy expenditure per ion pair in gases and gas mixtures, Rep. Prog. Phys., 21, 1-29, 1958.

Vroom, D. A., and F. J. De Heer, Production of exicted atoms by impact of fast electrons on molecular hydrogen and deuterium, $J$. Chem. Phys., 50, 580-590, 1969.

Waite, J. H., T. E. Cravens, J. V. Kozyra, R. H. Chen, S. K. Atreya, and A. F. Nagy, Aeronomical effects of the Jovian aurora, submitted to J. Geophys. Res., 1982.

Weaver, L. D., and R. H. Hughes, Production of $n=3$ and $n=4$ states of atomic hydrogen by electron impact on $\mathrm{H}_{2}, J$. Chem. Phys., 52, 2299-2301, 1970.

Yung, Y. L., and D. F. Strobel, Hydrocarbon photochemistry and Lyman alpha albedo of Jupiter, Astrophys. J., 239, 395-402, 1980.

(Received August 4, 1981; revised January 27, 1982; accepted February 2, 1982.) 